\begin{tabular}{|c|c|c|c|c|c|c|c|c|}
\hline \multicolumn{9}{|c|}{2008 MRS FALL MEETING SESSION LOCATOR } \\
\hline \multirow[t]{2}{*}{ SYMP. } & \multirow[t]{2}{*}{ TITLE } & \multirow[t]{2}{*}{ LOCATION } & \multicolumn{3}{|c|}{ MONDAY, DECEMBER 1} & \multicolumn{3}{|c|}{ TUESDAY, DECEMBER 2} \\
\hline & & & a.m. & p.m. & eve. ${ }^{*}$ & a.m. & p.m. & eve. ${ }^{*}$ \\
\hline A & $\begin{array}{l}\text { Performance \& } \\
\text { Reliability of Semi- } \\
\text { conductor Devices } \\
\text { Tutorial }^{* *} \\
\end{array}$ & $\begin{array}{l}\text { Room } 202 \\
\text { (Hynes) }\end{array}$ & $\begin{array}{l}\text { A1: GaN Devices } \\
\text { A2: GaN Device } \\
\text { Reliability }\end{array}$ & $\begin{array}{l}\text { A3: GaN Transistors } \\
\text { A4: GaN Material } \\
\text { Development }\end{array}$ & & \begin{tabular}{|l} 
A5: Nano Devices \\
A6: Optoelectronics
\end{tabular} & $\begin{array}{l}\text { A7: SiC Devices } \\
\text { A8: THz \& Graphene } \\
\text { Devices }\end{array}$ & A9: Posters \\
\hline B & $\begin{array}{l}\text { Transparent Conductors } \\
\text { \& Semiconductors for } \\
\text { Optoelectronics }\end{array}$ & $\begin{array}{l}\text { Room } 203 \\
\text { (Hynes) }\end{array}$ & $\begin{array}{l}\text { B1: TCO Fundamental } \\
\text { Electronic Structure }\end{array}$ & $\begin{array}{l}\text { 32: Unconventional } \\
\text { Transparent } \\
\text { Conductors }\end{array}$ & B3: Posters & \begin{tabular}{|l|} 
B4: Amorphous Oxide \\
Semiconductors I
\end{tabular} & $\begin{array}{l}\text { B5: Amorphous Oxide } \\
\text { Semiconductors II }\end{array}$ & B6: Posters \\
\hline $\mathbf{C}$ & $\begin{array}{l}\text { Theory \& Applications } \\
\text { of Ferroelectric \& } \\
\text { Multiferroic Materials } \\
\text { Tutorial }^{\star \star}\end{array}$ & $\begin{array}{l}\text { Room 210 } \\
\text { (Hynes) }\end{array}$ & $\begin{array}{l}\text { C1: Multiferroics- } \\
\text { Fundamentals }\end{array}$ & $\begin{array}{l}\text { 22: Multiferroics- } \\
\text { Surface \& Interface } \\
\text { Phenomena }\end{array}$ & C3: Posters & $\begin{array}{l}\text { C4/L1: Magnetoelectric } \\
\text { Coupling in } \\
\text { Multiferroics }\end{array}$ & $\begin{array}{l}\text { C5: BiFeO3- } \\
\text { Ferroelectric \& } \\
\text { Magnetic Properties }\end{array}$ & C6: Posters \\
\hline $\mathbf{D}$ & $\begin{array}{l}\text { Rare-Earth Doping of } \\
\text { Advanced Materials for } \\
\text { Photonic Applications }\end{array}$ & $\begin{array}{l}\text { Room } 201 \\
\text { (Hynes) }\end{array}$ & & \begin{tabular}{|l|} 
D1: Mechanisms \& \\
Laser Materials
\end{tabular} & & D2: Nitrides I & D3: Nitrides II & D4: Posters \\
\hline $\mathbf{E}$ & 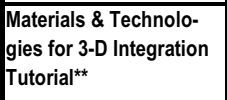 & $\begin{array}{l}\text { Room 205 } \\
\text { (Hynes) }\end{array}$ & \begin{tabular}{|l|} 
E1: Intro \& \\
Applications
\end{tabular} & E2: 3-D Bonding & & E3: TSV \& Thinning & $\begin{array}{l}\text { E4: 3-D Packaging \& } \\
\text { Epi 3-D }\end{array}$ & E5: Posters \\
\hline $\mathbf{F}$ & \begin{tabular}{|l|} 
Low-Cost Solution- \\
Based Deposition of \\
Inorganic Films for \\
Electronic/Photonic \\
Devices
\end{tabular} & $\begin{array}{l}\text { Room } 208 \\
\text { (Hynes) }\end{array}$ & & & & $\begin{array}{l}\text { F1: Nano-Related } \\
\text { Deposition }\end{array}$ & $\begin{array}{l}\text { F2: Solution-Processed } \\
\text { Electronic/Optical Films } \\
\text { \& Devices }\end{array}$ & F3: Posters \\
\hline $\mathbf{G}$ & $\begin{array}{l}\text { Organic \& Hybrid } \\
\text { Materials for Large-Area } \\
\text { Functional Systems }\end{array}$ & $\begin{array}{c}\text { Room } 207 \\
\text { (Hynes) }\end{array}$ & $\begin{array}{l}\text { G1: Conformal } \\
\text { Macroelectronics } \\
\text { G2: Macroelectronics } \\
\text { on Flexible Substrates }\end{array}$ & \begin{tabular}{|l|} 
G3: Large-Area \\
Compatible Processes \\
\& Materials I \\
G4: Materials \& \\
Systems for Emissive \\
Large-Area Displays
\end{tabular} & G5: Posters & \begin{tabular}{|l|}
$\begin{array}{l}\text { G6: Organic Materials } \\
\text { for Macroelectronics }\end{array}$ \\
\end{tabular} & \begin{tabular}{|l} 
G7: Device \& \\
Materials Physics
\end{tabular} & G8: Posters \\
\hline $\mathbf{H}$ & $\begin{array}{l}\text { Physics \& Technology } \\
\text { of Organic Semicon- } \\
\text { ductor Devices }\end{array}$ & $\begin{array}{c}\text { Room } 311 \\
\text { (Hynes) }\end{array}$ & & & & $\begin{array}{l}\text { H1/QQ5: Organic } \\
\text { Photovoltaic Cells }\end{array}$ & $\begin{array}{l}\text { H2: Organic Crystals, } \\
\text { Strong Coupling, \& } \\
\text { Spin Dynamics }\end{array}$ & \\
\hline $\mathbf{I}$ & \begin{tabular}{|l|} 
Reliability \& Properties \\
of Electronic Devices on \\
Flexible Substrates
\end{tabular} & $\begin{array}{c}\text { Room } 209 \\
\text { (Hynes) }\end{array}$ & \begin{tabular}{|l|}
$11:$ Flexible \\
Electronics
\end{tabular} & \begin{tabular}{|l} 
12: Flexible Displays \\
13: Processes on \\
Flexible Substrates I
\end{tabular} & & $\begin{array}{l}\text { 14: Metallic Thin Films } \\
\text { 15: Thin Films on } \\
\text { Flexible Substrates }\end{array}$ & $\begin{array}{l}\text { 16: Processes on } \\
\text { Flexible Substrates II } \\
\text { 17: Flexible Optical } \\
\text { Devices } \\
\end{array}$ & 18: Posters \\
\hline $\mathbf{J}$ & $\begin{array}{l}\text { Material Science for } \\
\text { Quantum Information } \\
\text { Processing } \\
\text { Technologies }\end{array}$ & $\begin{array}{c}\text { Room } 308 \\
\text { (Hynes) }\end{array}$ & J1: Semiconductors I & J2: Semiconductors II & J3: Posters & J4: Semiconductors III & J5: Semiconductors IV & \\
\hline $\mathbf{K}$ & $\begin{array}{l}\text { Magnetic Nano- } \\
\text { structures by Design }\end{array}$ & $\begin{array}{c}\text { Room } 206 \\
\text { (Hynes) }\end{array}$ & \begin{tabular}{|l|} 
K1: Magnetic Thin \\
Films \& Multilayers
\end{tabular} & $\begin{array}{l}\text { K2: Magnetic } \\
\text { Nanoparticles I }\end{array}$ & & $\begin{array}{l}\text { K3: Magnetic } \\
\text { Nanoparticles II }\end{array}$ & \begin{tabular}{|l} 
K4: Patterned Thin \\
Films
\end{tabular} & \\
\hline $\mathbf{L}$ & $\begin{array}{l}\text { New Materials with High } \\
\text { Spin Polarization \& } \\
\text { Their Applications }\end{array}$ & $\begin{array}{c}\text { Room } 204 \\
\text { (Hynes) }\end{array}$ & & & & $\begin{array}{l}\text { L1/C4: Magnetoelectric } \\
\text { Coupling in } \\
\text { Multiferroics } \\
\text { (Room 210-H) }\end{array}$ & $\begin{array}{l}\text { L2: New Half-Metallic } \\
\text { Compounds, Heusler } \\
\text { Compounds, Magnetic } \\
\text { Tunnel Junctions with } \\
\text { Half-Metallic } \\
\text { Ferromagnets }\end{array}$ & \\
\hline $\mathbf{M}$ & $\begin{array}{l}\text { Energy Harvesting- } \\
\text { Molecules \& Materials }\end{array}$ & $\begin{array}{l}\text { Republic A } \\
\text { (Sheraton) }\end{array}$ & M1: Dye Sensitization & $\begin{array}{l}\text { M2: OLED Materials } \\
\text { M3: Solar Cell } \\
\text { Polymers }\end{array}$ & M4: Posters & $\begin{array}{l}\text { M5: Energy Transfer } \\
\text { Processes } \\
\text { M6: Supramolecular } \\
\text { Systems }\end{array}$ & $\begin{array}{l}\text { M7: Charge Transfer } \\
\text { Processes } \\
\text { M8: Piezoelectrics }\end{array}$ & \\
\hline $\mathbf{N}$ & $\begin{array}{l}\text { Next-Generation \& } \\
\text { Nanoarchitectured } \\
\text { Photovoltaics } \\
\text { Tutorial }^{* *}\end{array}$ & $\begin{array}{l}\text { Republic B } \\
\text { (Sheraton) }\end{array}$ & \begin{tabular}{|l} 
N1: Multiple Exciton \\
Generation \& Electron \\
Transport \\
N2: Multiple Exciton \\
Generation \& \\
Nanocrystal Thin-Film \\
Devices
\end{tabular} & \begin{tabular}{|l|} 
N3: Carbon \\
Nanotubes-Carrier \\
Multiplication \& \\
Photovoltaics \\
N4: CdSe \& CdTe \\
Nanocrystals- \\
Synthesis, Properties, \\
\& Devices
\end{tabular} & & $\begin{array}{l}\text { N5: Intermediate } \\
\text { Band Solar Cells } \\
\text { N6: Up/Down } \\
\text { Conversion \& } \\
\text { Intermediate Band } \\
\text { Solar Cells }\end{array}$ & $\begin{array}{l}\text { N7: Plasmonics in } \\
\text { Photovoltaics } \\
\text { N8: Photon } \\
\text { Management \& Si } \\
\text { Quantum Dot Solar } \\
\text { Cells }\end{array}$ & N9: Posters \\
\hline $\mathbf{O}$ & $\begin{array}{l}\text { Structure/Property } \\
\text { Relationships in } \\
\text { Fluorite-Derivative } \\
\text { Compounds }\end{array}$ & $\begin{array}{l}\text { Fairfax B } \\
\text { (Sheraton) }\end{array}$ & & & & $\begin{array}{l}\text { 01: Fluorites-General } \\
\text { Structure/Property } \\
\text { Relationships I }\end{array}$ & \begin{tabular}{|l|} 
O2: Fluorites-General \\
Structure/Property \\
Relationships II
\end{tabular} & 03: Posters \\
\hline I & $\begin{array}{l}\text { Ssions: All E } \\
\text { Tutorial Sche }\end{array}$ & g Pos & $\begin{array}{l}\text { essions L } \\
\text { Shaded E }\end{array}$ & ocks: No & ition & Hynes) & & \\
\hline
\end{tabular}




\begin{tabular}{|c|c|c|c|c|c|c|c|c|}
\hline \multicolumn{9}{|c|}{2008 MRS FALL MEETING SESSION LOCATOR } \\
\hline SYMP. & \multicolumn{3}{|c|}{ WEDNESDAY, DECEMBER 3} & \multicolumn{3}{|c|}{ THURSDAY, DECEMBER 4} & \multicolumn{2}{|c|}{ FRIDAY, DECEMBER 5} \\
\hline & a.m. & p.m. & eve $^{*}$ & a.m. & p.m. & eve $^{*}$ & a.m. & p.m. \\
\hline A & $\begin{array}{l}\text { A10: Dielectrics } \\
\text { A11: Advanced Devices }\end{array}$ & \begin{tabular}{|l|} 
A12: Advanced \\
Materials \\
A13: Thin Film Transistors
\end{tabular} & & & & & & \\
\hline B & B7: ZnO Materials & $\begin{array}{l}\text { B8: ZnO Spintronics } \\
\text { \& P-Type ZnO }\end{array}$ & & $\begin{array}{l}\text { B9: Nanotube TCs \& } \\
\text { Atomic Layer Deposition }\end{array}$ & $\begin{array}{l}\text { B10:Towards } \\
\text { Amorphous Oxide } \\
\text { Electronics }\end{array}$ & & & \\
\hline $\mathbf{C}$ & $\begin{array}{l}\text { C7: Strain-Induced } \\
\text { Ferroelectricity }\end{array}$ & \begin{tabular}{l|} 
C8N10: Device \\
Applications of Multiferroics
\end{tabular} & c9: Posters & \begin{tabular}{|l|} 
C10: Novel Multiferroic \\
Materials \& \\
Heterostructures
\end{tabular} & $\begin{array}{l}\text { C11: Polarization } \\
\text { Catastrophe } \\
\text { C12: Novel } \\
\text { Characterization } \\
\text { Techniques }\end{array}$ & & $\begin{array}{l}\text { C13: Domain } \\
\text { Structure \& Dynamics } \\
\text { (Republic B-S) }\end{array}$ & \\
\hline $\mathbf{D}$ & $\begin{array}{l}\text { D5: Rare Earth } \\
\text { Doping \& Devices in } \\
\text { Silicon-Related Materials }\end{array}$ & $\begin{array}{l}\text { D6/MM9: Er-Doped Si } \\
\text { Nanostructures } \\
\text { (Room 309-H) }\end{array}$ & D7: Posters & $\begin{array}{l}\text { D8: Phosphors \& } \\
\text { Scintillators }\end{array}$ & & & & \\
\hline $\mathbf{E}$ & $\begin{array}{l}\text { E6: Materials \& } \\
\text { Modeling }\end{array}$ & & & & & & & \\
\hline $\mathbf{F}$ & $\begin{array}{l}\text { F4: Porous \& Structured } \\
\text { Films }\end{array}$ & $\begin{array}{l}\text { F5: Printing,Lithography, } \\
\text { Patteming }\end{array}$ & F6: Posters & $\begin{array}{l}\text { F7/P6: Solution } \\
\text { Processed Photovoltaic } \\
\text { Materials }\end{array}$ & \begin{tabular}{|l} 
F8: Bath-Based \\
Deposition \& Liquid \\
Precursor Routes
\end{tabular} & F9: Posters & & \\
\hline $\mathbf{G}$ & $\begin{array}{l}\text { G9: From Devices to } \\
\text { Circuits \& Systems I } \\
\text { G10: Energy Generation } \\
\text { \& Storage I }\end{array}$ & $\begin{array}{l}\text { G11: Solution-Processed } \\
\text { Nanomaterials }\end{array}$ & G12: Posters & \begin{tabular}{|l|} 
G13: Large-Area \\
Compatible Processing \\
\& Materials II \\
\end{tabular} & $\begin{array}{l}\text { G14: From Devices to } \\
\text { Circuits \& Systems II } \\
\text { G15: Energy } \\
\text { Generation \& Storage } \\
\text { II }\end{array}$ & & & \\
\hline $\mathbf{H}$ & $\begin{array}{l}\text { H3: Organic } \\
\text { Transitors }\end{array}$ & $\begin{array}{l}\text { H4: Organic Light- } \\
\text { Emitting Devices }\end{array}$ & H5: Posters & \begin{tabular}{|l|} 
H6: Organic \\
Photovoltaics I
\end{tabular} & $\begin{array}{l}\text { H7: Organic } \\
\text { Photovoltaics II }\end{array}$ & H8: Posters & $\begin{array}{l}\text { H9: Organic Interfaces- } \\
\text { Fundamentals \& Devices } \\
\text { (Independence West-S) }\end{array}$ & \\
\hline I & \begin{tabular}{|l|} 
|9: Small-Scale \\
Mechanical Properties I
\end{tabular} & & & & & & & \\
\hline $\mathbf{J}$ & \begin{tabular}{|l|} 
J6: Semiconductors V \\
J7: Superconductors I
\end{tabular} & J8: Superconductors II & & & & & & \\
\hline $\mathbf{K}$ & \begin{tabular}{|l} 
K5: Correlated \\
Materials
\end{tabular} & \begin{tabular}{|l|} 
K6: Spintronic \\
Applications
\end{tabular} & K: Posters & $\begin{array}{l}\text { K8: Magnetic } \\
\text { Characterization }\end{array}$ & $\begin{array}{l}\text { K9: Magnetic } \\
\text { Nanowires \& Other } \\
\text { Nanostructures }\end{array}$ & & & \\
\hline $\mathbf{L}$ & $\begin{array}{l}\text { L3: Theoretical Studies of } \\
\text { Half-Metallic Behavior in } \\
\text { Bulk Materials \& Interfaces }\end{array}$ & $\begin{array}{l}\text { L4: Applications of } \\
\text { Half-Metallic Ferro- } \\
\text { magnets, Including Shape } \\
\text { Memory, Nanoparticles, } \\
\text { CPP-GMR, Spin Filter, \& } \\
\text { Spin Dynamics }\end{array}$ & L5: Posters & $\begin{array}{l}\text { L6: Half-Metallic Oxides } \\
\text { \& New Ferromagnetic } \\
\text { Semiconductors, e.g., } \\
\text { Double Perovskites, } \\
\text { Doped ZnO, Spinels, } \\
\text { etc. }\end{array}$ & $\begin{array}{l}\text { 7: Advanced } \\
\text { Characterization } \\
\text { Methods, Including } \\
\text { Spin Polarization } \\
\text { Measurement }\end{array}$ & & & \\
\hline $\mathbf{M}$ & $\begin{array}{l}\text { M9: Quantum Dots } \\
\text { M10: Nanostructures }\end{array}$ & & & & & & & \\
\hline $\mathbf{N}$ & $\begin{array}{l}\text { N10: Epitaxial Quantum } \\
\text { Dot \& Quantum Well Solar } \\
\text { Cells } \\
\text { N11: Hot Carrier \& } \\
\text { Epitaxial Quantum Dot } \\
\text { Solar Cells }\end{array}$ & $\begin{array}{l}\text { 112: Luminescent Solar } \\
\text { Concentrators } \\
\text { N13: Nanowire Solar Cells } \\
\end{array}$ & N14: Posters & \begin{tabular}{|l|} 
N15: Type II B\& Offset \\
Nanostructures \& \\
Related Materials \\
N16: Advances in Dye- \\
Sensitized Solar Cells \& \\
Photocatalysis
\end{tabular} & $\begin{array}{l}\text { N17: Inorganic } \\
\text { Absorber Sensitized } \\
\text { Solar Cells } \\
\text { N18: Inorganic/ } \\
\text { Organic Hybrid Solar } \\
\text { Cells }\end{array}$ & & & \\
\hline $\mathbf{O}$ & $\begin{array}{l}\text { O4: Fluorites-Electromag- } \\
\text { netic \& Optical Properties } \\
\text { o5: Fluorites-lon } \\
\text { Transport I }\end{array}$ & $\begin{array}{l}\text { 06: Fluorites-lon } \\
\text { Transport II } \\
\text { 07/Q6: Fluorites-Actinide } \\
\text { Fuel \& Waste Forms } \\
\text { (Back Bay D-S) }\end{array}$ & & $\begin{array}{l}\text { O8: Fluorites-Radiation } \\
\text { Effects I } \\
\text { O9: Fluorites-Radiation } \\
\text { Effects II }\end{array}$ & & & & \\
\hline
\end{tabular}




\begin{tabular}{|c|c|c|c|c|c|c|c|c|}
\hline \multicolumn{9}{|c|}{2008 MRS FALL MEETING SESSION LOCATOR } \\
\hline \multirow[t]{2}{*}{ SYMP. } & \multirow[t]{2}{*}{ TITLE } & \multirow[t]{2}{*}{ LOCATION } & \multicolumn{3}{|c|}{ MONDAY, DECEMBER 1} & \multicolumn{3}{|c|}{ TUESDAY, DECEMBER 2} \\
\hline & & & a.m. & p.m. & eve.* & a.m. & p.m. & eve. ${ }^{*}$ \\
\hline $\mathbf{P}$ & $\begin{array}{l}\text { Photovoltaic Materials \& } \\
\text { Manufacturing Issues }\end{array}$ & $\begin{array}{l}\text { Independence East } \\
\text { (Sheraton) }\end{array}$ & & & & $\begin{array}{l}\text { P1: Si-Based Materials, } \\
\text { Solar Cells } \\
\text { Manufacturing }\end{array}$ & $\begin{array}{l}\text { P2: Optical Effect, } \\
\text { Light Trapping, } \\
\text { Crystallization }\end{array}$ & \\
\hline $\mathbf{Q}$ & $\begin{array}{l}\text { Scientific Basis for } \\
\text { Nuclear Waste } \\
\text { Management XXXII }\end{array}$ & $\begin{array}{l}\text { Back Bay D } \\
\text { (Sheraton) }\end{array}$ & $\begin{array}{l}\text { Q1: National Programs } \\
\text { \& Advanced Fuel } \\
\text { Cycles }\end{array}$ & $\begin{array}{l}\text { Q2: Spent Nuclear } \\
\text { Fuel }\end{array}$ & & $\begin{array}{l}\text { Q3: Nuclear Waste } \\
\text { Glasses \& Vitrification }\end{array}$ & $\begin{array}{l}\text { Q4: Ceramic } \\
\text { Wasteforms }\end{array}$ & \\
\hline $\mathbf{R}$ & $\begin{array}{l}\text { Materials for Future } \\
\text { Fusion \& Fission } \\
\text { Technologies }\end{array}$ & $\begin{array}{l}\text { Independence West } \\
\text { (Sheraton) }\end{array}$ & & & & $\begin{array}{l}\text { R1: ODS } \\
\text { R2: Structural Materials }\end{array}$ & \begin{tabular}{|l} 
R3: Defects \\
R4: Fundamental \\
Aspects
\end{tabular} & \\
\hline $\mathbf{S}$ & Solid-State lonics & $\begin{array}{l}\text { Back Bay A } \\
\text { (Sheraton) }\end{array}$ & $\begin{array}{l}\text { S1: Micro SOFCs- } \\
\text { From Materials to } \\
\text { Devices }\end{array}$ & $\begin{array}{l}\text { S2: Modelling \& } \\
\text { Fundamental Studies }\end{array}$ & S3: Posters & $\begin{array}{l}\text { S4: Innovative } \\
\text { Concepts for Energy } \\
\text { Storage } \\
\text { S5: Battery Materials- } \\
\text { Electrolytes \& Systems }\end{array}$ & $\begin{array}{l}\text { S6/PP5: Solid State } \\
\text { lonics for Energy } \\
\text { (Back Bay C-S) }\end{array}$ & \\
\hline $\mathbf{T}$ & Mobile Energy & $\begin{array}{c}\text { Liberty } \\
\text { (Sheraton) }\end{array}$ & T1: Batteries I & $\begin{array}{l}\text { T2: Cathodes \& } \\
\text { Anodes }\end{array}$ & & T3: Batteries II & $\begin{array}{l}\text { T4: Novel Conversion } \\
\text { \& Storage Techniques }\end{array}$ & T5: Posters \\
\hline $\mathbf{U}$ & $\begin{array}{l}\text { Advanced Intermetallic- } \\
\text { Based Alloys for } \\
\text { Extreme Environment \& } \\
\text { Energy Applications }\end{array}$ & $\begin{array}{l}\text { Constitution A } \\
\text { (Sheraton) }\end{array}$ & $\begin{array}{l}\text { U1: Intermetallics for } \\
\text { Hydrogen Storage \& } \\
\text { Thermoelectric } \\
\text { Applications }\end{array}$ & $\begin{array}{l}\text { U2: Iron Aluminides- } \\
\text { Physical Metallurgy, } \\
\text { Processing, \& } \\
\text { Properties }\end{array}$ & & \begin{tabular}{|l} 
U3: Titanium \\
Aluminides $1-$ \\
Physical Metallurgy, \\
Processing, \& \\
Properties
\end{tabular} & $\begin{array}{l}\text { U: Titanium } \\
\text { Aluminides II- } \\
\text { Structure, Properties, } \\
\text { \& Coatings }\end{array}$ & U5: Posters \\
\hline $\mathbf{V}$ & $\begin{array}{l}\text { Materials, Devices, \& } \\
\text { Characterization for } \\
\text { Smart Systems }\end{array}$ & $\begin{array}{c}\text { Commonwealth } \\
\text { (Sheraton) }\end{array}$ & $\begin{array}{l}\text { V1: Magnetostrictives } \\
\text { V2: Multifunctionals \& } \\
\text { Multiferroics }\end{array}$ & $\begin{array}{l}\text { V3: Sensors \& Novel } \\
\text { Processing }\end{array}$ & V4: Posters & $\begin{array}{l}\text { V5: Novel Active } \\
\text { Materials-Polymers } \\
\text { V6: Novel Active } \\
\text { Materials-Composites }\end{array}$ & V7: Optics & \\
\hline $\mathbf{W}$ & \begin{tabular}{|l|} 
Computational Materials \\
Design via Multiscale \\
Modeling \\
Tutorial $^{\star \star}$
\end{tabular} & $\begin{array}{c}\text { Constitution B } \\
\text { (Sheraton) }\end{array}$ & $\begin{array}{l}\text { W1: New Approaches } \\
\text { Toward Multiscale } \\
\text { Materials Design }\end{array}$ & $\begin{array}{l}\text { W2: Materials in } \\
\text { Energy Applications }\end{array}$ & & $\begin{array}{l}\text { W3: Nano Technology } \\
\text { \& Devices }\end{array}$ & $\begin{array}{l}\text { W4: Hetrogenous } \\
\text { Materials } \\
\text { W5: Polymer \& } \\
\text { Biomaterials }\end{array}$ & W6: Posters \\
\hline $\mathbf{X}$ & $\begin{array}{l}\text { Frontiers of Materials } \\
\text { Research }\end{array}$ & $\begin{array}{c}\text { Grand Ballroom } \\
\text { (Sheraton) }\end{array}$ & & $\mathrm{x} 1$ & & & $\mathrm{x} 2$ & \\
\hline $\mathbf{Y}$ & $\begin{array}{l}\text { Biomineral Interfaces- } \\
\text { From Experiment to } \\
\text { Theory }\end{array}$ & $\begin{array}{c}\text { Berkeley A/B } \\
\text { (Sheraton) }\end{array}$ & $\begin{array}{l}\text { Y1: The Organic/ } \\
\text { Mineral Interface }\end{array}$ & $\begin{array}{l}\text { Y2: Biomolecules on } \\
\text { Surfaces }\end{array}$ & Y3: Posters & $\begin{array}{l}\text { Y4: Mesocrystals } \\
\text { \& Aggregation }\end{array}$ & $\begin{array}{l}\text { Y5: Biomimetics \& } \\
\text { Biomineralization }\end{array}$ & \\
\hline $\mathbf{Z}$ & $\begin{array}{l}\text { Mechanics of Biological } \\
\text { \& Bioreplacement } \\
\text { Materials }\end{array}$ & $\begin{array}{l}\text { Back Bay B } \\
\text { (Sheraton) }\end{array}$ & & & & \begin{tabular}{|l} 
Z1: Tissue \\
Mechanics I
\end{tabular} & $\begin{array}{l}\text { Z2: Tissue } \\
\text { Mechanics II }\end{array}$ & \\
\hline AA & $\begin{array}{l}\text { Materials for Optical } \\
\text { Sensors in Biomedical } \\
\text { Applications }\end{array}$ & $\begin{array}{l}\text { Gardner A/B } \\
\text { (Sheraton) }\end{array}$ & & $\begin{array}{l}\text { AA1: Biomedical } \\
\text { Devices for Resources } \\
\text { Limited Setting } \\
\text { AA2: Waveguide-based } \\
\text { Sensors }\end{array}$ & & $\begin{array}{l}\text { AA3: Interferometric } \\
\text { Biosensors } \\
\text { AA4: Defraction } \\
\text { Biosensors }\end{array}$ & $\begin{array}{l}\text { AA5: Fluorescence- } \\
\text { based Biosensors } \\
\text { AA6: Surface Sensing }\end{array}$ & AA7: Posters \\
\hline BB & $\begin{array}{l}\text { Polymer-Based Smart } \\
\text { Materials-Process, } \\
\text { Properties, \& } \\
\text { Application }\end{array}$ & $\begin{array}{l}\text { Grand Ballroom } \\
\text { (Sheraton) }\end{array}$ & & & & $\begin{array}{l}\text { BB1: New Materials } \\
\text { \& Characterization }\end{array}$ & $\begin{array}{l}\text { BB2: Device } \\
\text { Application }\end{array}$ & \\
\hline CC & $\begin{array}{l}\text { Design, Fabrication, \& } \\
\text { Self Assembly of } \\
\text { "Patchy" \& Anisometric } \\
\text { Particles }\end{array}$ & $\begin{array}{c}\text { Fairfax A } \\
\text { (Sheraton) }\end{array}$ & & & & $\begin{array}{l}\text { CC1: Self Assembly } \\
\text { of Anisotropic Particles }\end{array}$ & $\begin{array}{l}\text { CC2: Synthesis of } \\
\text { Patchy \& Anisometric } \\
\text { Particles }\end{array}$ & CC3: Posters \\
\hline DD & $\begin{array}{l}\text { Materials in Tissue } \\
\text { Engineering }\end{array}$ & $\begin{array}{l}\text { Hampton A/B } \\
\text { (Sheraton) }\end{array}$ & $\begin{array}{l}\text { DD1: Novel Materials } \\
\text { in Tissue Engineering }\end{array}$ & $\begin{array}{l}\text { DD2: Cell-Responsive } \\
\text { Materials }\end{array}$ & DD3/HH6: Posters & $\begin{array}{l}\text { DD4: Applied Tissue } \\
\text { Engineering-Tissue } \\
\text { Engineered Products \& } \\
\text { Materials Issues in } \\
\text { Industry }\end{array}$ & $\begin{array}{l}\text { DD5: Scafold } \\
\text { Fabrication Methods } \\
\end{array}$ & \\
\hline EE & $\begin{array}{l}\text { Nano- \& Microscale } \\
\text { Materials-Mechanical } \\
\text { Properties \& Behavior } \\
\text { under Extreme } \\
\text { Environments }\end{array}$ & $\begin{array}{c}\text { Room 200 } \\
\text { (Hynes) }\end{array}$ & \begin{tabular}{|l} 
EE1/NN1 In-situ \\
Nanomechanics
\end{tabular} & $\begin{array}{l}\text { EE2: Anomalous } \\
\text { Nanomechanical } \\
\text { Behavior }\end{array}$ & & $\begin{array}{l}\text { EE3: Fatigue, Fracture, } \\
\& \text { Tribology }\end{array}$ & $\begin{array}{l}\text { EE4: Thin Films, } \\
\text { Multilayers, \& } \\
\text { Nanocomposites- } \\
\text { Mechanics \& Radiation } \\
\text { Effects }\end{array}$ & EE5: Posters \\
\hline FF & $\begin{array}{l}\text { Nanofunctional } \\
\text { Materials, Structures, \& } \\
\text { Devices for Bio-medical } \\
\text { Applications } \\
\text { Tutorial }{ }^{\star \star}\end{array}$ & $\begin{array}{c}\text { Room } 304 \\
\text { (Hynes) }\end{array}$ & $\begin{array}{l}\text { Tutorial } \\
\text { FF1: Nanotechnology } \\
\text { for Biomedical } \\
\text { Applications }\end{array}$ & $\begin{array}{l}\text { FF2: Nanoparticles for } \\
\text { Drug Delivery }\end{array}$ & FF3: Posters & \begin{tabular}{|l|} 
FF4: In-vitro \\
Diagnostics \\
Using Nanodevices
\end{tabular} & $\begin{array}{l}\text { FF5: Nanodevices for } \\
\text { Screening \& Analysis }\end{array}$ & FF6: Posters \\
\hline GG & $\begin{array}{l}\text { Microelectromechanical } \\
\text { Systems-Materials \& } \\
\text { Devices II }\end{array}$ & $\begin{array}{c}\text { Room } 306 \\
\text { (Hynes) }\end{array}$ & $\begin{array}{l}\text { GG1: Materials \& } \\
\text { Processes for MEMS }\end{array}$ & $\begin{array}{l}\text { GG2: Microdevices } \\
\text { \& Micro/Nanofluidics }\end{array}$ & GG3: Posters & $\begin{array}{l}\text { GG4: Micro/ } \\
\text { Nanomechanics }\end{array}$ & $\begin{array}{l}\text { GG5: MEMS } \\
\text { Reliability \& Tribology }\end{array}$ & \\
\hline f & $\begin{array}{l}\text { essions: All Ev } \\
\text { Tutorial Sche }\end{array}$ & ling Post & $\begin{array}{r}\text { Sessions Lo } \\
\text { Shaded BI }\end{array}$ & $\begin{array}{l}\text { cated in Ext } \\
\text { ocks: No Se }\end{array}$ & $\begin{array}{l}\text { ibition H } \\
\text { ssion }\end{array}$ & Hynes) & & \\
\hline
\end{tabular}




\section{MRS FALL MEETING SESSION LOCATOR}

\begin{tabular}{|c|c|c|c|c|c|c|c|c|}
\hline \multicolumn{9}{|c|}{2008 MRS FALL MEETING SESSION LOCATOR } \\
\hline SYMP. & \multicolumn{3}{|c|}{ WEDNESDAY, DECEMBER 3} & \multicolumn{3}{|c|}{ THURSDAY, DECEMBER 4} & \multicolumn{2}{|c|}{ FRIDAY, DECEMBER 5} \\
\hline & a.m. & p.m. & eve* & a.m. & p.m. & eve $^{*}$ & a.m. & p.m. \\
\hline $\mathbf{P}$ & $\begin{array}{l}\text { P3: Hetrojunction Solar } \\
\text { Cells, Solar Cell } \\
\text { Processing, Poly- } \\
\text { crystalline Silicon }\end{array}$ & P4: Organic Solar Cells & P5: Posters & $\begin{array}{l}\text { P6/F7: Solution- } \\
\text { Processed } \\
\text { Photovoltaic Materials } \\
\text { (Room 208-H) }\end{array}$ & \begin{tabular}{|l|} 
77: Thin-Film \\
Materials, Thin-Film \\
Solar Materials, Thin- \\
Film Solar Cells, \\
Processing
\end{tabular} & & & \\
\hline $\mathbf{Q}$ & $\begin{array}{l}\text { Q5: Engineered } \\
\text { Barrier Systems, the Near } \\
\text { Field \& Cementitious }\end{array}$ & $\begin{array}{l}\text { Q6/07: Fluorites- } \\
\text { Actinide Fuel, \& Waste } \\
\text { Forms }\end{array}$ & Q7: Posters & $\begin{array}{l}\text { Q8: Focus on YUCCA } \\
\text { Mountain }\end{array}$ & $\begin{array}{l}\text { Q9: Container } \\
\text { Corrosion }\end{array}$ & Q10: Posters & $\begin{array}{l}\text { Q11: Migration } \\
\text { \& Colloids }\end{array}$ & \\
\hline $\mathbf{R}$ & R5: Radiation Damage & R6: Coatings & R7: Posters & $\begin{array}{l}\text { R8: Defect Evolution } \\
\text { R9: Fuels }\end{array}$ & \begin{tabular}{|l|} 
R10: Fuels \\
Modeling
\end{tabular} & & & \\
\hline $\mathbf{S}$ & $\begin{array}{l}\text { S7/T6: Solid State lonics } \\
\text { for Mobile Energy }\end{array}$ & $\begin{array}{l}\text { S8: Cathodes for } \\
\text { SOFCs }\end{array}$ & S9: Posters & $\begin{array}{l}\text { S10: Solid Oxide } \\
\text { Fuel Cells I }\end{array}$ & $\begin{array}{l}\text { S11: Solid Oxide Fuel } \\
\text { Cells II } \\
\text { S12: PEM Fuel Cells }\end{array}$ & S13: Posters & $\begin{array}{l}\text { S14: Battery } \\
\text { Materials-Electrodes }\end{array}$ & \\
\hline $\mathbf{T}$ & $\begin{array}{l}\text { T6/S7: Solid State lonics } \\
\text { for Mobile Energy } \\
\text { (Back Bay A-S) }\end{array}$ & T7: Fuel Cells & & & & & & \\
\hline $\mathbf{U}$ & $\begin{array}{l}\text { U6: Nickel/Cobalt } \\
\text { Superalloys, \& Nickel } \\
\text { Aluminides }\end{array}$ & $\begin{array}{l}\text { U7: Niobium \& Molyb- } \\
\text { denum Silicide-Based } \\
\text { Alloys }\end{array}$ & & $\begin{array}{l}\text { U8: Laves Phases- } \\
\text { Structure \& Properties }\end{array}$ & $\begin{array}{l}\text { U9: Fundamental } \\
\text { Aspects of Inter- } \\
\text { metallics-Phase } \\
\text { Stability, Defects, } \\
\text { Theory }\end{array}$ & & & \\
\hline $\mathbf{V}$ & $\begin{array}{l}\text { V8: Actuators \& Energy } \\
\text { Conversion } \\
\text { V9: Thin Film } \\
\text { Ferroelectrics }\end{array}$ & $\begin{array}{l}\text { V10/C8: Device } \\
\text { Applications of Multiferroics } \\
\text { (Room 210-H) }\end{array}$ & V11: Posters & $\begin{array}{l}\text { V12: Shape Memory } \\
\text { V13: Ferromagnetic } \\
\text { Shape Memory }\end{array}$ & V14: MEMS & & & \\
\hline $\mathbf{W}$ & $\begin{array}{l}\text { W7: Metals \& Alloys- } \\
\text { From Atom to } \\
\text { Microstructure I }\end{array}$ & $\begin{array}{l}\text { W8: Metals \& Alloys- } \\
\text { From Atom to } \\
\text { Microstructure II } \\
\text { W9: Thin Film \& Coating }\end{array}$ & & $\begin{array}{l}\text { EE8/W10: Computa- } \\
\text { tional Nanomechanics } \\
\text { I-Dislocations \& } \\
\text { Radiation Effects }\end{array}$ & \begin{tabular}{|l|} 
W11/EE9: Computa- \\
tional Nanomechanics \\
II-Nanocrystals \& \\
Nanowires
\end{tabular} & W12: Posters & $\begin{array}{l}\text { W13: Computational } \\
\text { Mechanics }\end{array}$ & \\
\hline $\mathbf{X}$ & & $\mathrm{x} 3$ & & & $\mathrm{X} 4$ & & & \\
\hline $\mathbf{Y}$ & & & & & & & & \\
\hline $\mathbf{Z}$ & Z3: Tissue Mechanics IV & Z4: Cellular Mechanics & & $\begin{array}{l}\text { 75: Mechanics of } \\
\text { Biomolecules }\end{array}$ & \begin{tabular}{|l|} 
Z6: Mechanics of \\
Biomedical Materials I
\end{tabular} & Z7, Z8: Posters & $\begin{array}{l}\text { Z9: Mechanics of } \\
\text { Biomedical Materials II }\end{array}$ & \\
\hline $\mathbf{A A}$ & $\begin{array}{l}\text { AA8: Label-Free Sensors } \\
\text { for Complex Fluid I }\end{array}$ & $\begin{array}{l}\text { AA9: Surface-Enhanced } \\
\text { Raman } \\
\text { AA10: Label-Free Sensors } \\
\text { for Complex Fluid II }\end{array}$ & & & & & & \\
\hline BB & $\begin{array}{l}\text { BB3: New Materials } \\
\text { \& Characterization }\end{array}$ & BB4: Device Application & BB5: Posters & $\begin{array}{l}\text { BB6: New Materials } \\
\text { \& Characterization I }\end{array}$ & $\begin{array}{l}\text { BB7: New Materials } \\
\text { \& Characterization II }\end{array}$ & BB8: Posters & $\begin{array}{l}\text { BB9: E-NSF \& New } \\
\text { Materials }\end{array}$ & \\
\hline CC & $\begin{array}{l}\text { CC4: Theory \& } \\
\text { Simulation of Self } \\
\text { Assembly }\end{array}$ & $\begin{array}{l}\text { CC5: Fabrication \& } \\
\text { Assembly I }\end{array}$ & & $\begin{array}{l}\text { CC6: Fabrication } \\
\text { \& Assembly II }\end{array}$ & & & & \\
\hline DD & & & & & & & & \\
\hline EE & $\begin{array}{l}\text { EE6: Deformation } \\
\text { Mechanisms at Small- } \\
\text { Length Scales }\end{array}$ & \begin{tabular}{|l|} 
EE7: Nanomechanical \\
Testing \& Characterization
\end{tabular} & & $\begin{array}{l}\text { EE8/W10: Computa- } \\
\text { tional Nanomechanics } \\
\text { l-Dislocations \& } \\
\text { Radiation Effects } \\
\text { (Constitution B-S) }\end{array}$ & \begin{tabular}{|l|} 
EE9/W11: Computa- \\
tional Nanomechanics \\
$11-$ Nanocrystals \& \\
Nanowires \\
(Constitution B-S)
\end{tabular} & EE10: Posters & \begin{tabular}{|l|} 
EE11: Deformation \\
under Shock, High Strain \\
Rate, \& High Pressure \\
Conditions \\
(Liberty-S)
\end{tabular} & \\
\hline FF & $\begin{array}{l}\text { FF7: Multifunctional } \\
\text { Nanoparticles for } \\
\text { Biomedical Applications }\end{array}$ & $\begin{array}{l}\text { FF8: Interfacial Surface } \\
\text { Functionalization \& } \\
\text { Dynamics for Biomedical } \\
\text { Applications }\end{array}$ & FF9: Posters & $\begin{array}{l}\text { FF10: Nanomaterial } \\
\text { Formation \& } \\
\text { Interactions for } \\
\text { Biomedical Applications }\end{array}$ & $\begin{array}{l}\begin{array}{l}\text { FF11: Nanoparticles } \\
\text { for In-vivo Bioimaging }\end{array} \\
\end{array}$ & FF12: Posters & & \\
\hline $\mathbf{G G}$ & & & & & & & & \\
\hline
\end{tabular}




\begin{tabular}{|c|c|c|c|c|c|c|c|c|}
\hline \multicolumn{9}{|c|}{2008 MRS FALL MEETING SESSION LOCATOR } \\
\hline \multirow[t]{2}{*}{ SYMP. } & \multirow[t]{2}{*}{ TITLE } & \multirow[t]{2}{*}{ LOCATION } & \multicolumn{3}{|c|}{ MONDAY, DECEMBER 1} & \multicolumn{3}{|c|}{ TUESDAY, DECEMBER 2} \\
\hline & & & a.m. & p.m. & eve.* & a.m. & p.m. & eve. ${ }^{*}$ \\
\hline $\mathbf{H H}$ & \begin{tabular}{|l|} 
Advances in Material \\
Design for Regenerative \\
Medicine, Drug Delivery, \\
\& Targeting/lmaging
\end{tabular} & $\begin{array}{l}\text { Room } 310 \\
\text { (Hynes) }\end{array}$ & $\begin{array}{l}\text { HH1: Advanced } \\
\text { Biomaterials I } \\
\text { HH2: Drug Delivery } \\
\text { Systems I }\end{array}$ & \begin{tabular}{|l} 
HH3: Advanced \\
Biomaterials II \\
HH4: Drug Delivery \\
Systems II-Gene \& \\
Peptide Delivery
\end{tabular} & $\begin{array}{l}\text { HH5: Posters } \\
\text { HH6/DD3: Posters }\end{array}$ & $\begin{array}{l}\text { HH7: Honoring Prof. } \\
\text { Robert Langer I } \\
\text { (Ballroom A-H) }\end{array}$ & $\begin{array}{l}\text { HH8: Honoring Prof. } \\
\text { Robert Langer II } \\
\text { (Ballroom A-H) }\end{array}$ & \\
\hline II & $\begin{array}{l}\text { Bio-inspired } \\
\text { Transduction, } \\
\text { Fundamentals, \& } \\
\text { Applications }\end{array}$ & $\begin{array}{l}\text { Room } 301 \\
\text { (Hynes) }\end{array}$ & & & & \begin{tabular}{|l|} 
|11: Sensors for \\
Biomedical Applications
\end{tabular} & \begin{tabular}{|l|}
112 : Synthesis \& \\
Characterization of Bio- \\
inspired Materials
\end{tabular} & 113: Posters \\
\hline JJ & $\begin{array}{l}\text { Nanotubes, Nano- } \\
\text { wires, Nanobelts, \& } \\
\text { Nanocoils-Promise, } \\
\text { Expectations, \& Status }\end{array}$ & $\begin{array}{l}\text { Room } 302 \\
\text { (Hynes) }\end{array}$ & \begin{tabular}{|l|}
$J J 1:$ Carbon Nanotube \\
Synthesis \\
JJ2: Carbon Nanotube \\
Growth Mechanisms
\end{tabular} & $\begin{array}{l}\text { JJ3: Carbon Nanotube } \\
\text { Processing } \\
\text { JJ4: Solution-Based } \\
\text { Processing \& } \\
\text { Electrochemistry }\end{array}$ & JJ5: Posters & $\begin{array}{l}\text { JJ6: Graphene- } \\
\text { Synthesis } \\
\text { JJ7: Graphene } \\
\text { Characterization }\end{array}$ & \begin{tabular}{|l|} 
JJ8: Nanowires- \\
Synthesis \& \\
Characterization I \\
Jjg: Nanowires- \\
Synthesis \& \\
Characterization II
\end{tabular} & JJ10: Posters \\
\hline KK & $\begin{array}{l}\text { Transport Properties in } \\
\text { Polymer Nano- } \\
\text { composites }\end{array}$ & $\begin{array}{l}\text { Room } 300 \\
\text { (Hynes) }\end{array}$ & $\begin{array}{l}\text { KK1: Composites for } \\
\text { Renewable Energy }\end{array}$ & \begin{tabular}{|l} 
KK2: Smart Polymer \\
Nanocomposites
\end{tabular} & & $\begin{array}{l}\text { KK3: Electrically } \\
\text { Conductive Polymer } \\
\text { Nanocomposites }\end{array}$ & $\begin{array}{l}\text { KK4: Nanocomposite } \\
\text { Membranes }\end{array}$ & KK5: Posters \\
\hline LL & $\begin{array}{l}\text { Nanowires-Synthesis, } \\
\text { Properties, Assembly, } \\
\text { \& Application }\end{array}$ & $\begin{array}{l}\text { Room } 312 \\
\text { (Hynes) }\end{array}$ & $\begin{array}{l}\text { LL1: Growth } \\
\text { Mechanisms }\end{array}$ & $\begin{array}{l}\text { LL2: Advances in } \\
\text { Growth \& } \\
\text { Characterization }\end{array}$ & LL3, LL4: Posters & $\begin{array}{l}\text { LL5: Doping } \\
\text { LL6: Device } \\
\text { Applications-Logic \& } \\
\text { Memory }\end{array}$ & $\begin{array}{l}\text { LL7: Emerging } \\
\text { Application }\end{array}$ & \\
\hline MM & $\begin{array}{l}\text { Applications of Group } \\
\text { IV Semiconductor } \\
\text { Nanostructures }\end{array}$ & $\begin{array}{c}\text { Room } 309 \\
\text { (Hynes) }\end{array}$ & \begin{tabular}{|l|} 
MM1: Light Emission \\
\& Photonic Devices
\end{tabular} & $\begin{array}{l}\text { MM2: SiGe } \\
\text { Nanostructures II } \\
\text { MM3: Si } \\
\text { Nanostructures I }\end{array}$ & MM4: Posters & \begin{tabular}{|l|} 
MM5: Si \& Ge \\
Nanocrystals
\end{tabular} & MM6: Diamondoids & \\
\hline NN & $\begin{array}{l}\text { In-situ Studies across } \\
\text { Spatial \& Temporal } \\
\text { Length Scales for } \\
\text { Nanoscience \& } \\
\text { Technology } \\
\text { Tutorial }{ }^{* *}\end{array}$ & $\begin{array}{l}\text { Room } 102 \\
\text { (Hynes) }\end{array}$ & $\begin{array}{l}\text { NN1/EE1: In-situ } \\
\text { Nanomechanics } \\
\text { (Room 200-H) }\end{array}$ & $\begin{array}{l}\text { NN2: In situ Growth } \\
\& \text { Characterization of } \\
\text { Nanotubes }\end{array}$ & NN3: Posters & \begin{tabular}{|l|} 
NN4: Nucleation, \\
Growth, \& Coarsening \\
Kinetics of \\
Nanostructures
\end{tabular} & $\begin{array}{l}\text { NN5: Ultrafast } \\
\text { Microscopy \& } \\
\text { Diffraction }\end{array}$ & NN6: Posters \\
\hline 00 & $\begin{array}{l}\text { Grazing-Incidence Small } \\
\text { Angle X-Ray Scattering }\end{array}$ & $\begin{array}{l}\text { Room } 104 \\
\text { (Hynes) }\end{array}$ & & & & $\begin{array}{l}\text { 001: GISAXS Studies } \\
\text { of Block Copolymer \& } \\
\text { Polymer Thin Films I }\end{array}$ & \begin{tabular}{|l|} 
OO2: GISAXS Studies \\
of Block Copolymer \& \\
Polymer Thin Films II
\end{tabular} & 003: Posters \\
\hline PP & $\begin{array}{l}\text { Solid-State Chemistry of } \\
\text { Inorganic Materials VII }\end{array}$ & $\begin{array}{l}\text { Back Bay C } \\
\text { (Sheraton) }\end{array}$ & $\begin{array}{l}\text { PP1: Novel Synthetic } \\
\text { Methods }\end{array}$ & $\begin{array}{l}\text { PP2: Fe-As } \\
\text { Superconductors \& } \\
\text { Beyond }\end{array}$ & PP3: Posters & $\begin{array}{l}\text { PP4: Synthesis, } \\
\text { Crystal Chemistry, \& } \\
\text { Physical Properties of } \\
\text { Oxides } \\
\end{array}$ & $\begin{array}{l}\text { PP5/S6: Solid-State } \\
\text { lonics for Energy }\end{array}$ & \\
\hline $\mathbf{Q Q}$ & $\begin{array}{l}\text { Synthesis \& Processing } \\
\text { of Organic \& Polymeric } \\
\text { Functional Materials for } \\
\text { a Sustainable Energy } \\
\text { Economy }\end{array}$ & $\begin{array}{l}\text { Room } 313 \\
\text { (Hynes) }\end{array}$ & \begin{tabular}{|l|} 
QQ1:Conjugated \\
Polymers-Synthesis \& \\
Processing \\
QQ2: Materials \& \\
Devices Processing
\end{tabular} & $\begin{array}{l}\text { QQ3: OLED I- } \\
\text { Materials \& Devices }\end{array}$ & QQ4: Posters & $\begin{array}{l}\text { QQ5/H1: Organic } \\
\text { Photovoltaic Cells } \\
\text { (Room 311-H) }\end{array}$ & \begin{tabular}{|l|} 
QQ6: Photovoltaics I- \\
Materials \& Devices for \\
Vapor-Deposited Solar \\
Cells \\
QQ7: Photovoltaics \\
II-Materials \& Devices \\
for Polymer-based \\
Solar Cells \\
\end{tabular} & QQ8: Posters \\
\hline $\mathbf{R R}$ & $\begin{array}{l}\text { Artificially Induced } \\
\text { Grain Alignment in Thin } \\
\text { Films }\end{array}$ & $\begin{array}{c}\text { Room } 111 \\
\text { (Hynes) }\end{array}$ & & & & $\begin{array}{l}\text { RR1: Milestones in } \\
\text { IBAD Texturing } \\
\text { RR2: IBAD Texturing }\end{array}$ & \begin{tabular}{|l|} 
RR3: IBAD Materials \& \\
Applications
\end{tabular} & RR4: Posters \\
\hline SS & $\begin{array}{l}\text { Selecting \& Qualifying } \\
\text { New Materials for Use in } \\
\text { Regulated Industries }\end{array}$ & $\begin{array}{l}\text { Room } 306 \\
\text { (Hynes) }\end{array}$ & & & & & & \\
\hline TT & $\begin{array}{l}\text { Local Structure \& } \\
\text { Dynamics in } \\
\text { Amorphous } \\
\text { Systems } \\
\text { Tutorial } \\
\end{array}$ & $\begin{array}{l}\text { Room } 103 \\
\text { (Hynes) }\end{array}$ & \begin{tabular}{|l|} 
TT1: Structure of \\
Liquids \& Glasses
\end{tabular} & \begin{tabular}{|l|} 
TT2: Frans Spaepen \\
Colloquium
\end{tabular} & TT3: Posters & $\begin{array}{l}\text { TT4: Dynamics- } \\
\text { Relaxation \& Crystal } \\
\text { Nucleation }\end{array}$ & $\begin{array}{l}\text { TT5: Atomistic } \\
\text { Modeling } \\
\text { TT6: Reactions \& } \\
\text { Crystallization }\end{array}$ & TT7: Posters \\
\hline ( & $\begin{array}{l}\text { essions: All Ev } \\
\text { Tutorial Schec }\end{array}$ & ng Pos & $\begin{array}{l}\text { Sessions LC } \\
\text { Shaded B }\end{array}$ & $\begin{array}{l}\text { cated in Ex } \\
\text { ocks: No S }\end{array}$ & $\begin{array}{l}\text { oition r } \\
\text { sion }\end{array}$ & Hynes) & & \\
\hline
\end{tabular}




\section{MRS FALL MEETING SESSION LOCATOR}

\begin{tabular}{|c|c|c|c|c|c|c|c|c|}
\hline \multicolumn{9}{|c|}{2008 MRS FALL MEETING SESSION LOCATOR } \\
\hline SYMP. & WEDI & VESDAY, DECEMBE & R 3 & THUR & SDAY, DECEMB & ER 4 & FRIDAY, DE & IBER 5 \\
\hline & a.m. & p.m. & eve $^{*}$ & a.m. & p.m. & eve $^{*}$ & a.m. & p.m. \\
\hline $\mathrm{HH}$ & $\begin{array}{l}\text { HH9: Tissue Engineering } \\
\text { HH10: Controlling Stem } \\
\text { Cell Function }\end{array}$ & $\begin{array}{l}\text { HH11: Advanced Materials } \\
\text { for Imaging } \\
\text { HH12: Cancer Targeting }\end{array}$ & HH13: Posters & & & & & \\
\hline II & $\begin{array}{l}\text { 114: Applications of Bio- } \\
\text { inspired Materials }\end{array}$ & |115: Bio-inspired Sensors & & & & & & \\
\hline JJ & $\begin{array}{l}\text { JJ11: Electronic \& Optical } \\
\text { Properties I } \\
\text { JJ12: Electronic \& Optical } \\
\text { Properties II }\end{array}$ & $\begin{array}{l}\text { JJ13: Optical Properties \& } \\
\text { Raman Spectroscopy } \\
\text { JJ14: Optical \& Thermal } \\
\text { Properties }\end{array}$ & Jง15: Posters & $\begin{array}{l}\text { JJ16: Mechanical } \\
\text { Properties I } \\
\text { JJ17: Mechanical } \\
\text { Properties II }\end{array}$ & $\begin{array}{l}\text { JJ18: Nanocomposites } \\
\text { JJ19: Device } \\
\text { Applications }\end{array}$ & JJ20: Posters & & \\
\hline KK & $\begin{array}{l}\text { KK6: Microstructure } \\
\text { KK7: Thermally } \\
\text { Conductive } \\
\text { Nanocomposites }\end{array}$ & & & & & & & \\
\hline LL & $\begin{array}{l}\text { LL8: Electro/ } \\
\text { Mechanical Properties } \\
\text { LL9: Electrical \& Thermal } \\
\text { Transport }\end{array}$ & $\begin{array}{l}\text { LL10: Sensing } \\
\text { LL11: Advances in Growth } \\
\text { \& Characterization III }\end{array}$ & LL12,13: Posters & $\begin{array}{l}\text { LL14: Synthesis of } \\
\text { Heterostructures } \\
\text { LL15: Optical Properties } \\
\text { I }\end{array}$ & \begin{tabular}{|l|} 
LL16: Optical \\
Properties II-Alloys \& \\
Heterostructures \\
LL17: Photodetection
\end{tabular} & $\begin{array}{l}\text { LL18, LL19, } \\
\text { LL20: Posters }\end{array}$ & \begin{tabular}{|l}
$L$ 21: Metal \\
Nanowires \\
(Constitution A-S)
\end{tabular} & \\
\hline MM & $\begin{array}{l}\text { MM7: Ultrathin Si Layers } \\
\text { MM8: Si Nanostructures II }\end{array}$ & $\begin{array}{l}\text { MM9/D6: Er-Doped } \\
\text { Si Nanostructures }\end{array}$ & & $\begin{array}{l}\text { MM10: Si Devices } \\
\text { MM11: Si } \\
\text { Nanostructures III }\end{array}$ & $\begin{array}{l}\text { MM12: Nanowires \& } \\
\text { Thermolelectrics } \\
\text { MM13: Porous Silicon } \\
\text { \& Beyond }\end{array}$ & & & \\
\hline NN & $\begin{array}{l}\text { NN7: Kinetics of Phase } \\
\text { Transformations in } \\
\text { Nanomaterials }\end{array}$ & $\begin{array}{l}\text { NN8: Low-Energy } \\
\text { Electron \& X-ray } \\
\text { Microscopies }\end{array}$ & NN9: Psoters & $\begin{array}{l}\text { NN10: In-situ Studies } \\
\text { of Thin-Film Growth }\end{array}$ & \begin{tabular}{|l|} 
NN11: In-situ \\
Scanning Probe \\
Microscopy Studies of \\
Surface Structures \& \\
Properties
\end{tabular} & & & \\
\hline 00 & $\begin{array}{l}\text { OO4: GISAXS Studies of } \\
\text { Nanoparticle \& } \\
\text { Nanoporous Thin Films }\end{array}$ & $\begin{array}{l}\text { OO5: GISAXS } \\
\text { Studies of Biomolecular } \\
\text { Materials, Complex \& } \\
\text { Structured Interfaces }\end{array}$ & & $\begin{array}{l}\text { O06: GISAXS } \\
\text { Studies of Inorganic } \\
\text { Thin Film \& } \\
\text { Nanostructured Growth }\end{array}$ & & & & \\
\hline PP & $\begin{array}{l}\text { PP6: Synthesis, } \\
\text { Properties, \& } \\
\text { Characterization of } \\
\text { Nanomaterials }\end{array}$ & $\begin{array}{l}\text { PP7: Nanostructures \& Thin } \\
\text { Films }\end{array}$ & PP8, PP9: Posters & \begin{tabular}{|l} 
PP10: Porous \& \\
Framework Materials
\end{tabular} & \begin{tabular}{|l|} 
PP11: Thermoelectrics \\
\& Spintronics
\end{tabular} & $\begin{array}{l}\text { PP12, PP13, PP14: } \\
\text { Posters }\end{array}$ & \begin{tabular}{l|} 
PP15: Characterization \\
of Complex Structures
\end{tabular} & \\
\hline QQ & $\begin{array}{l}\text { QQ9: Photovoltaics III- } \\
\text { Organic-lnorganic Hybrid } \\
\text { Solar Cells } \\
\text { QQ10: Photovoltaics IV- } \\
\text { Materials \& Devices for } \\
\text { Polymer-based Solar Cells }\end{array}$ & $\begin{array}{l}\text { QQ11: Photovoltaics V- } \\
\text { Device Engineering \& } \\
\text { Physics } \\
\text { QQ12: Interface } \\
\text { Engineering \& Physics }\end{array}$ & & & & & & \\
\hline $\mathbf{R R}$ & $\begin{array}{l}\text { RR5: IBAD Long-Length } \\
\text { Application } \\
\text { RR6: Texturing by } \\
\text { Sputtering \& Other } \\
\text { Techniques } \\
\end{array}$ & $\begin{array}{l}\text { RR7: Inclined Substrate } \\
\text { Deposition \& Vicinal } \\
\text { Substrates }\end{array}$ & & & & & & \\
\hline SS & $\begin{array}{l}\text { SS1: Qualifying New } \\
\text { Materials I } \\
\text { SS2: In-Room Poster } \\
\text { Session I }\end{array}$ & $\begin{array}{l}\text { SS3: Qualifying New } \\
\text { Materials II }\end{array}$ & & & & & & \\
\hline TT & $\begin{array}{l}\text { TT8: Beyond Metallic } \\
\text { Systems } \\
\text { TT9: Deformation of } \\
\text { Metallic Glasses I }\end{array}$ & $\begin{array}{l}\text { TT10: Deformation } \\
\text { of Metallic Glasses II }\end{array}$ & & & & & & \\
\hline
\end{tabular}

